\title{
USPSTF expands options for cervical cancer screening
}

\section{Women ages 30 to 65 years now have a third cervical cancer screening option.}

\section{PRACTICE CHANGER}

Offer women ages 30 to 65 years the option of being screened for cervical cancer using a high-risk human papillomavirus assay every 5 years. $^{1,2}$

STRENGTH OF RECOMMENDATION

A: Based on a US Preventive Services Task Force recommendation statement.

Curry SJ, Krist AH, Owens DK, et al. Screening for cervical cancer: US Preventive Services Task Force Recommendation Statement. JAMA. 2018;320:674-686.

\section{ILLUSTRATIVE CASE}

A 35-year-old healthy woman without a history of high-grade precancerous cervical lesions, immunodeficiency, or exposure to diethylstilbestrol presents to your office for her routine health visit. During your conversation with her, she shares, "I read on the Internet that I only need to be tested for human papillomavirus, but I'm wondering how I'll be checked for cervical cancer." She asks for your opinion about cervical cancer screening methods.

$\mathrm{T}$ he National Cancer Institute predicts that there will be 13,800 new cases of cervical cancer this year, with an estimated 4290 deaths. $^{3}$ This type of cancer is primarily caused by high-risk human papillomavirus (hrHPV) infections. Fortunately, high-grade precancerous cervical lesions and cervical cancer can be detected with routine Papanicolaou (Pap) smears, which have led to a substantial decrease in the number of deaths from cervical cancer in the United States-from 2.8 per 100,000 women in 2000 to 2.3 deaths per 100,000 women in $2015 .{ }^{3}$ In addition to hrHPV infection, risk factors for cervical cancer include low socioeconomic status, cigarette smoking, marrying before 18 years of age, young age at first coitus, multiple sexual partners, multiple sexual partners of a partner, and multiple childbirths. ${ }^{4}$

Cervical cancer is associated with numerous negative outcomes, including a decrease in quality of life, decreased libido, poor mental health, infertility, negative body image, and death. ${ }^{5}$ This is particularly true among women of lower socioeconomic status or whose language differs from that of their primary health care provider. ${ }^{1,5}$

Given the enormous impact cervical cancer screening has made on the detection and mortality rate of this devastating disease, ${ }^{4,5}$ it is crucial to identify the types of screening tests and screening intervals that lead to the greatest benefit and least harm for all patient populations. The US Preventive Services Task Force (USPSTF) previously addressed this issue in 2012, concluding that cytology alone every 3 years for women ages 21 to 65 years and cytology alone every 3 years or co-testing with cytology and hrHPV every 5 years in women ages 30 to 65 years was of substantial benefit (strength of recommendation [SOR]: A) ${ }^{6}$

\section{STUDY SUMMARY}

Another option for some women: hrHPV testing alone every 5 years

In this 2018 systematic review and modeling study by the USPSTF, randomized con-
Sonia Oyola, MD; Emily White VanGompel, MD, MPH

Department of Family Medicine, University of Chicago (Drs. Oyola and VanGompel); NorthShore University HealthSystem, Evanston, IL (Dr. VanGompel)

DEPUTY EDITOR Rebecca Mullen, MD University of Colorado Family Medicine Residency, Denver 


\section{$>$}

Any 1 of 3 screening methods is adequately sensitive for detecting precancerous high-grade cervical lesions or cervical cancer in women ages 30 to 65 years. trolled trials (RCTs) and cohort studies that compared cytology to hrHPV testing alone or co-testing (cytology with hrHPV) were used to determine the optimal frequency of, and age group for, cervical cancer screening that would yield the least harm and the most benefit from each of these screening methods. ${ }^{7-9}$

Similar to the previous recommendation, the USPSTF found that screening women $<21$ years or > 65 years if previously adequately screened (defined as 3 consecutive negative screenings or 2 negative screenings within the past 10 years with the most recent being within the past 5 years) led to more harm than benefit. They therefore concluded that women in these age groups should not be screened routinely (SOR: D). The USPSTF also recommends against cervical cancer screening in women who have had a hysterectomy with removal of the cervix and who do not have a history of a high-grade precancerous lesion or cervical cancer (SOR: D).

However, for women ages 21 to 65 years, the USPSTF found that screening substantially reduces cervical cancer incidence and mortality, and that for women ages 21 to 29 years, screening every 3 years with cytology alone offers the best balance of benefits and harms (SOR: A). For women ages 30 to 65 years, the USPSTF recommends screening every 3 years with cytology alone or every 5 years with either primary hrHPV testing or co-testing (hrHPV with cytology) (SOR: A). The recommendations apply to all asymptomatic women with a cervix; exceptions include those with a history of a highgrade precancerous cervical lesion or cancer, in utero exposure to diethylstilbestrol, or a compromised immune system.

The change in this current set of recommendations by the USPSTF is the inclusion of screening with hrHPV alone every 5 years as an additional cervical cancer screening option for women ages 30 to 65 years. The decision to include this option was based largely on a decision analysis model commissioned by the USPSTF and reviewed along with clinical trials and cohort studies. The modeling studies found that both primary hrHPV testing alone and co-testing every 5 years prevented a similar number of cervical cancer cases and required a similar number of colposcopies.

Finally, the USPSTF emphasized that screening alone is not sufficient for the prevention of cervical cancer and that efforts should be made to create equitable access to follow-up of abnormal results and the provision of appropriate treatment. ${ }^{1,2}$

\section{WHAT'S NEW}

\section{When it comes to cervical cancer screening, 3 solid options now exist}

The previous USPSTF recommendation concluded that women ages 30 to 65 years should be screened with either cytology alone every 3 years or co-testing (cytology and hrHPV) every 5 years. This systematic review and modeling study concluded that any one of the stated screening methods would be adequately sensitive for detecting precancerous high-grade cervical lesions or cervical cancer: cytology every 3 years, primary hrHPV every 5 years, or co-testing every 5 years. ${ }^{7-9}$

\section{CAVEATS}

\section{No studies comparing hrHPV to co-testing and no meta-analysis}

No studies were found that directly compared primary hrHPV testing with co-testing. ${ }^{1}$ A meta-analysis could not be performed due to the methodological differences in RCTs and cohort studies reviewed. The new recommendation is unique in its reliance on modeling to simulate a direct comparison of these 2 screening methods.

\section{CHALLENGES TO IMPLEMENTATION}

\section{Getting the word out}

\section{and increasing comfort levels}

The principal challenge to implementation lies in practitioners' knowledge of this new recommendation and a possible low comfort level with ordering hrHPV testing alone. Patients will need to be engaged in shared decision-making to understand and make use of the 3 options.

ACKNOWLEDGEMENT

The PURLs Surveillance System was supported in part by Grant Number UL1RR024999 from the National Center 
For Research Resources, a Clinical Translational Science Award to the University of Chicago. The content is solely the responsibility of the authors and does not necessarily represent the official views of the National Center For Research Resources or the National Institutes of Health. Copyright $\odot$ 2020. The Family Physicians Inquiries Network.

\section{References}

1. Curry SJ, Krist AH, Owens DK, et al. Screening for cervical cancer: US Preventive Services Task Force Recommendation Statement. JAMA. 2018;320:674-686.

2. Melnikow J, Henderson JT, Burda BU, et al. Screening for cervical cancer with high-risk human papillomavirus testing: a systematic evidence review for the US Preventive Services Task Force. Evidence Synthesis No. 158. Rockville, MD: Agency for Healthcare Research and Quality; 2018.

3. National Cancer Institute. Cancer Stat Facts. Cervix uteri. https:// seer.cancer.gov/statfacts/. Accessed July 1, 2020.

4. Momenimovahed Z, Salehiniya H. Incidence, mortal- ity and risk factors of cervical cancer in the world. Biomed Res Ther. 2017;4:1795-1811.

5. Ashing-Giwa KT, Kagawa-Singer M, Padilla GV, et al. The impact of cervical cancer and dysplasia: a qualitative, multiethnic study. Psychooncology. 2004;13:709-728.

6. Moyer VA; US Preventive Services Task Force. Screening for cervical cancer: US Preventive Services Task Force recommendation statement. Ann Intern Med. 2012; 156:880-891.

7. Ronco G, Giorgi-Rossi P, Carozzi F, et al; New Technologies for Cervical Cancer Screening (NTCC) Working Group. Efficacy of human papillomavirus testing for the detection of invasive cervical cancers and cervical intraepithelial neoplasia: a randomized controlled trial. Lancet Oncol. 2010;11:249-257.

8. Ronco G, Giorgi-Rossi P Carozzi F, et al; New Technologies for Cervical Cancer Screening Working Group. Results at recruitment from a randomized controlled trial comparing human papillomavirus testing alone with conventional cytology as the primary cervical cancer screening test. J Natl Cancer Inst. 2008;100:492-501.

9. Ogilvie GS, van Niekerk DJ, Krajden M, et al. A randomized controlled trial of human papillomavirus (HPV) testing for cervical cancer screening: trial design and preliminary results (HPV FOCAL Trial). BMC Cancer. 2010;10:111. 